\title{
Dynamic Stability Analysis of a Circularly Tapered Rotating Beam Subjected to Axial Pulsating Load and Thermal Gradient under Various Boundary Conditions
}

\author{
Rashmita Parida \\ Department of Mechanical Engineering, College of Engineering and Technology, Bhubaneswar Techno Campus, \\ Ghatikia, Bhubaneswar, Orissa, India-751003
}

\author{
Pusparaj Dash \\ Department of Mechanical Engineering, VSSUT Burla, Sambalpur, Orissa, India-768018
}

\begin{abstract}
(Received 13 August 2013; accepted 8 October 2015)
The dynamic stability of a circularly tapered rotating beam subjected to a pulsating axial external excitation with thermal gradient was studied for all possible combinations of clamped, guided, pinned, fixed, and free boundary conditions. The equations of motion and associated boundary conditions were obtained using the extended Hamilton's principle. Then these equations of motion and the associated boundary conditions were non-dimensionalised. A set of Hill's equations were obtained from the non-dimensional equations of motion by the application of the extended Galerkin method. The zones of parametric instability were obtained using Saito-Otomi conditions. The effects of various boundary conditions, thermal gradient, taper, and rotational speed on the regions of parametric instability were investigated and presented through a series of graphs. The results reveal that increasing rotational speed and taper have stabilizing effects, whereas increasing thermal gradient has a destabilizing effect for all boundary conditions of the beam.
\end{abstract}

\section{NOMENCLATURE}

\begin{tabular}{ll}
$A(x), A(\xi)$ & Area of a generic section of the beam \\
$A_{1}$ & Cross sectional area at the end $x=l$ \\
$C_{0}$ & Hub radius \\
$c_{0}$ & Dimensionless hub radius, $=C_{0} / l$ \\
$d(x), d(\xi)$ & Diameter of a generic section of the beam \\
$d_{1}$ & Diameter at the end, $x=l$ \\
$E(x), E(\xi)$ & Young's modulus at a generic section \\
$E_{1}$ & Young's modulus at the end, $x=l$ \\
$I(x), I(\xi)$ & Moment of inertia at a generic section \\
$I_{1}$ & Moment of inertia at the end, $x=l$ \\
$l$ & Length of the beam \\
$m(\xi)$ & Mass distribution function \\
$P_{0}$ & Static axial load \\
$P_{1}$ & Dynamic axial load \\
$p(\tau)$ & Dimensionless load \\
$p_{0}$ & Dimensionless static axial load \\
$p_{1}$ & Dimensionless dynamic axial load \\
$S(\xi)$ & Moment of inertia distribution function \\
$T(\xi)$ & Elasticity modulus distribution function \\
$t$ & Time \\
$w(x, t)$ & Transverse deflection of the beam \\
$\gamma$ & Coefficient of thermal expansion of the \\
$\delta$ & beam material \\
$a^{*}$ & Thermal gradient parameter \\
$\eta$ & Diameter taper parameter \\
$\xi$ & Dimensionless transverse deflection, $=w / l$ \\
$\tau$ & Dimensionless length, $=x / l$ \\
$\rho$ & Dimensionless time, $=c t$ \\
& Density of the beam material \\
\hline &
\end{tabular}

$\Omega \quad$ Uniform angular velocity $\Omega$ of the beam about $z^{\prime}$-axis

$\Omega_{0} \quad$ Rotational speed parameter

$\omega \quad$ Excitation frequency

$\omega_{0}$ Dimensionless fundamental natural frequency

$\Theta \quad$ Non-Dimensional excitation frequency, $=\omega / c$

$\Psi_{0} \quad$ Reference temperature

$\Psi_{1} \quad$ Temperature at the end, $\xi=1$

\section{INTRODUCTION}

The stability analysis and dynamic behaviour of a rotating cantilever beam with axial orientation perpendicular to the axis of spin is very essential for its practical applications such as turbomachinery blades, rotor blades of helicopter, aircraft propellers, flexible appendages of spacecraft, satellite antennas, and robotic manipulators, to name a few. In some cases the structures have to operate under elevated temperatures. A linear relation is observed between the Young's modulus and the temperature of most engineering materials.

An ample number of publications are available regarding the design and analysis of rotating structures. The flexural vibrations of a rotating cantilever beam with a tip mass at the free end has been studied by Bhat. ${ }^{1}$ He proposed the beam characteristic orthogonal polynomials in the Rayleigh-Ritz method. Liu and $\mathrm{Yeh}^{2}$ have investigated the influence of restrained parameters on the Eigen frequencies of rotating uniform and non-uniform beams with a restrained base using Galerkin's method. The stability analysis of a rotating shaft due to pulsating torque applied at its end has been studied by Unger and 